\title{
Development of an Autonomous Surface Vessel for Use as a Drone Base Station
}

\author{
Murray Lawn, ${ }^{1 *}$ Akihiro Morinaga, ${ }^{1}$ and Ikuo Yamamoto ${ }^{2}$ \\ ${ }^{1}$ Division of Mechanical Science, Nagasaki University Graduate School, \\ 1-14, Bunkyo, Nagasaki City, Nagasaki 852-8521, Japan \\ ${ }^{2}$ Organization for Marine Science and Technology, Office for Research Initiatives and Development, \\ Nagasaki University, 1-14, Bunkyo, Nagasaki City, Nagasaki 852-8521, Japan
}

(Received November 18, 2020; accepted February 10, 2021)

Keywords: autonomous surface vessel, drone base station, coastal debris

The increasing presence of coastal debris is problematic in many parts of the world. Coastal cleanups are very time-consuming and expensive. Part of this cost involves the monitoring of debris washed ashore. An effective way to enable careful analysis of the amounts of types of debris is the use of drone-based photography. However, many coastlines are not easily accessible. Therefore, to monitor extensive coastlines for coastal debris, an autonomous surface vessel (ASV) is being developed for use as a drone base station. The concept is for the user to remotely guide the ASV out of any given harbor, and once in the open sea, the vessel proceeds autonomously on preprogrammed GPS routes to specified locations. At the specified locations, when the conditions for drone operation are appropriate, the drone is released and takes photographs of coastal areas before returning to the ASV for recharging. This technology is expected to have wide potential for both open sea and coastal monitoring and potential application in assisting search and rescue operations.

\section{Introduction}

Drones are increasingly used to provide cost-effective surveillance and monitoring of fixed sites and plants. ${ }^{(1,2)}$ However, in the case of surveillance and monitoring of extensive coastlines, drones are limited in a number of aspects. Typically, an operator needs to drive to a given site, from which the drone can be operated within a range up to about $7 \mathrm{~km}$ using a remote control unit. This can be extended by using mobile connections, whereby the range will be limited by the drone's battery and the mobile phone coverage of the area to be monitored. Hybrid drones $^{(3)}$ can extend the flight distance, and the use of preprogrammed GPS flight paths can make it possible for them to operate independent of mobile coverage. However, depending on the purpose of the monitoring, in order to preprogram flight routes and specific areas requiring imaging, prior visits to the target site by a live operator may be necessary.

An initiative called the Ikkaku (Sea Unicorn) project, detailed in the acknowledgements, has adopted a number of sub-projects to deal with increasing ocean-based debris. One team,

*Corresponding author: e-mail: lawnmj@nagasaki-u.ac.jp

https://doi.org/10.18494/SAM.2021.3192 
initially referred to as Coastal Cleanup using Satellites and Drones (CCSD) and more recently renamed "Debris Watchers", is focusing on providing an efficient mechanism to monitor levels of coastal debris. A more cost-efficient mechanism using a combination of coastal satellite images and fixed-camera and drone-based camera data, the correlation between these data sets, and $\mathrm{AI}$ is being evaluated and compared with current monitoring techniques, which typically involve sending people out to specific sites to estimate the amounts of types of debris.

In the case of the Tsushima Islands between Japan and Korea, a large amount of seaborne debris washes up on the coast each year. ${ }^{(4)}$ However, most of the coastline of Tsushima is not easily accessible. The debris can only be conveniently observed from the air or by boat. In the case of viewing by boat, the low viewing angle makes it very difficult to estimate the extent of the debris in many cases. Aerial photography provides excellent visualization of the extent of debris but is very expensive. With the advent of drones, it has become possible to significantly reduce the cost of aerial photography; however, as mentioned above, access to many coastal areas is difficult. Furthermore, drones are limited in range by their battery operation. Therefore, a low-cost boat-based base station for drones, referred to as an Autonomous Surface VesselDrone Base Station (ASV-DBS), has been proposed.

In light of the above situation, the development of a boat that can host a drone and operate at a level of autonomy appropriate to any given conditions is being developed. Progress to date is outlined in this paper.

\section{ASV-DBS}

The ASV-DBS is shown in Figs. 1 and 2 during trials in Shimabara (Nagasaki, Japan). A video of previous sea trials in Tsushima, Shimabara and a close-up of the hatch mechanism can be seen online. ${ }^{(5,6)}$ The ASV-DBS main specifications/system are outlined in Table 1.

The above ASV-DBS currently has local remote control using an RC unit (Fubata T1486). Although a mobile control system has been set up and tested in a workshop, it has yet to be tested in the open sea.

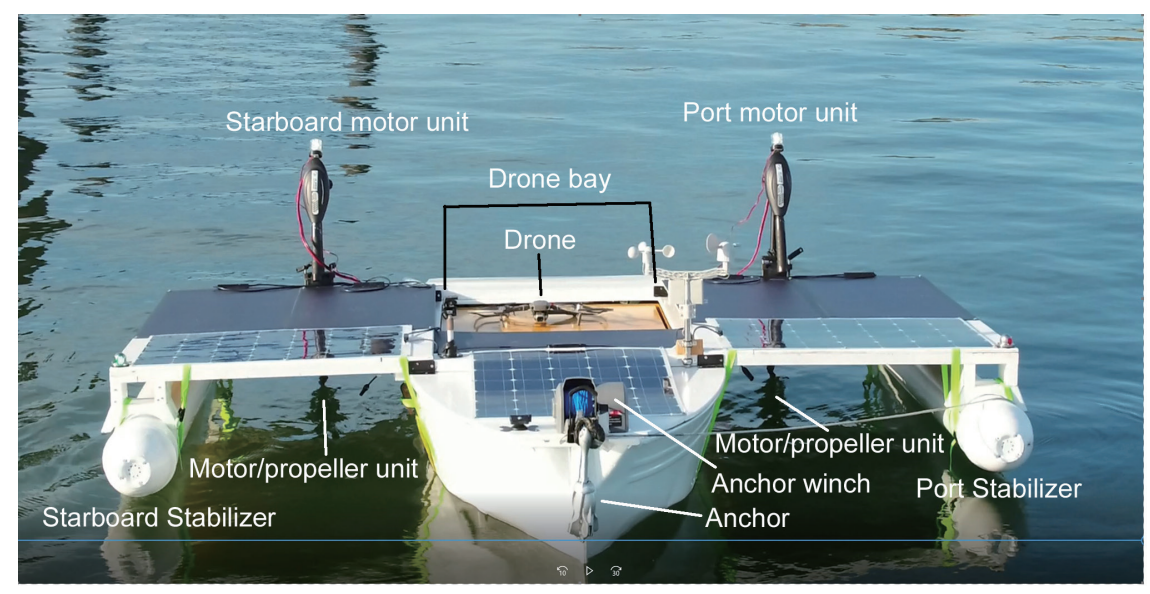

Fig. 1. (Color online) ASV-DBS (drone bay hatch open and raised). 


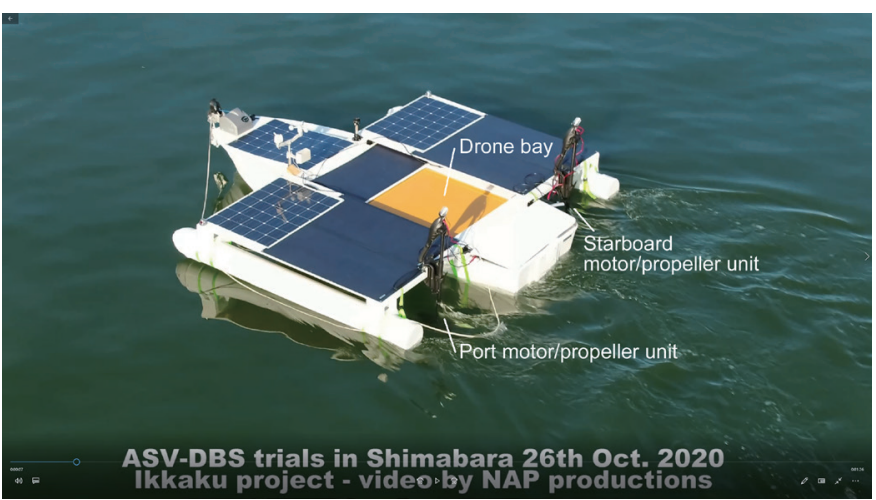

(a)

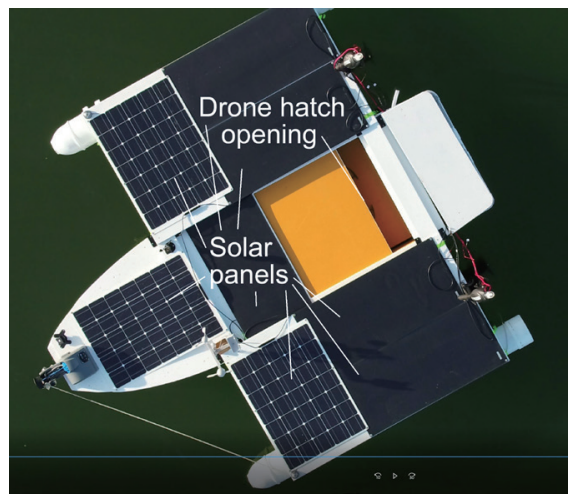

(b)

Fig. 2. (Color online) ASV-DBS drone bay lowered with (a) hatch closed and (b) hatch beginning to open.

Table 1

ASV-DBS—-specifications/systems.

\begin{tabular}{lc}
\hline Length & $3.3 \mathrm{~m}$ \\
\hline Width & $3.1 \mathrm{~m}$ with stabilizers, $1.1 \mathrm{~m}$ without \\
\hline Gross weight & $\sim 140 \mathrm{~kg}$ (including batteries) \\
\hline Motors & $600 \mathrm{~W} \times 2$ (limited to comply with $1 \mathrm{~kW}$ local AUV laws) \\
\hline Batteries & $2 \times \mathrm{M} 31 \mathrm{MF}(\sim 115 \mathrm{Ah}) 12 \mathrm{~V}$, weight $52 \mathrm{~kg}$ \\
\hline Solar panels & $8 \times \sim 100 \mathrm{~W}$ \\
\hline Main system controller & Pixhawk4 flight controller \\
\hline Main motor controller & Cytron Smart Drive Duo 60 \\
\hline Anchor winch & Modified Deckboat 40 Auto Deploy Anchor unit \\
\hline Weather unit & SwitchDoc Labs Weather plus2 (RP based) \\
\hline Radio control unit & Fubata T1486 for local control \\
\hline Mobile remote operation & $4 \mathrm{GPi}$ and RPi $4 \mathrm{~b}$ with fixed IP sim card using AUVcast-Pro ${ }^{(7)}$ working \\
\hline Depth monitor & with Mission Planner software ${ }^{(8)}$ \\
\hline Lighting & Sonar unit, Ping sonar altimeter and echo sounder ${ }^{(9)}$ \\
\hline Auto bilge pump & Full night time navigation lighting \\
\hline
\end{tabular}

\subsection{Proposed operation}

As mentioned above, as part of a coastal monitoring project, the long-term goal is to realize an ASV-DBS that can provide continuous monitoring and surveillance of Tsushima's extensive coastline $\left[\sim 915 \mathrm{~km}^{(10)}\right]$. In the case of monitoring sea debris on the Tsushima coastline, most of the hotspots are known from prior aerial photography, i.e., some areas tend to accumulate sea debris owing to their topography. Once the vessel is fully functional, we plan to have Tsushima Island circumnavigated by a regular boat, most likely a local fishing boat, with an ASV mission planning person working with a drone operator to preprogram ASV routes. This planning must establish the location of suitable areas for both anchoring overnight and performing drone photography, as well as optimal flight paths to and from the coastal areas requiring imaging, including the optimal flight altitude and clarification of the areas to be imaged. Prior to such 
operations, legal consent from all local and regional authorities is required, and the drone must not pass through or near no-go zones, such as areas of aquaculture. When aerially surveying coastal debris, our team has found from experience that an altitude of about $20 \mathrm{~m}$ provides an optimal balance between drone battery life and image quality that allows AI analysis of the debris. In the Ikkaku project, the Debris Watchers team includes a number of companies that specialize in drone operation, AI data analysis, and autonomous control. ${ }^{(11-13)}$

For long-term operation around Tsushima Island, the addition of a satellite communication link is proposed. This is necessary to provide the minimal essential data, which includes the current location and the occurrence of any problems that may require intervention. As mentioned above, most of this extensive coastline has very little road access or mobile coverage. In regard to data collection, it is proposed to provide wifi access at appropriate intervals by bringing the ASV to the entrance of the very few local harbors, anchoring it, and requesting remote assistance to guide the ASV into the harbor. From experience, most of the harbors can be problematic. In particular, after heavy rain or a typhoon, large amounts of soil, silt, stones, and rocks of various sizes are dumped into the harbors, making it necessary for a human operator to navigate the ASV using real-time camera, GPS, and sonar data to an area where long-term anchorage is possible and wifi access is available. This clearly needs arrangement in advance with each harbormaster/local administration. Access to wifi will facilitate the uploading of large amounts of image data to appropriate sites for AI analysis and direct observation, as well as provide the opportunity to check the preprogrammed settings and make any alterations as required. Currently, no collision avoidance system is provided. Although the use of Lidar is planned because the Pixhawk flight controller directly supports the use of Lidar for object avoidance, the Pixhawk system primarily assumes drone operation. The application of Lidar to ASV operation is detailed in Ref. 14.

\subsection{ASV-DBS main control unit}

The ASV-DBS control unit hardware is shown in Fig. 3. The drone hatch and lifter are powered by the driver board on the right (RC input-MDDS-10). Currently the mechanism is simply hard-wired with switches and diodes to provide a reliable drone release unit. When a given polarity is applied, the hatch slider opens, and once open, a switch on the hatch slider actuates the lifter until it is in the fully raised position; actuation is automatically stopped by a built-in limit switch on the linear actuator. When the polarity is reversed on the drone release unit, the lifter retracts, and a switch at the fully retracted position actuates the hatch slider to close, with actuation automatically stopped by an external switch on the slider when fully closed.

The drone lifter mechanism is configured similarly to most low-profile lifting platforms, powered by a commercial $12 \mathrm{~V}, 100 \mathrm{~mm}$ linear actuator, providing the platform $(86 \times 86 \mathrm{~cm})$ with $\sim 25 \mathrm{~cm}$ of lift (built-in limit switches are provided within the actuator unit). The hatch slider is powered by a $12 \mathrm{~V}$ motor via a flexible joint and bearing connected to a $12 \mathrm{~mm} \times \sim 1 \mathrm{~m}$ threaded rod. The rod is connected to the hatch (cover) via a $\sim 40 \mathrm{~mm}$ nut that moves along the length of the rod, and the other end is fixed to a bearing. External limit switches have been fitted. ${ }^{(6)}$ 


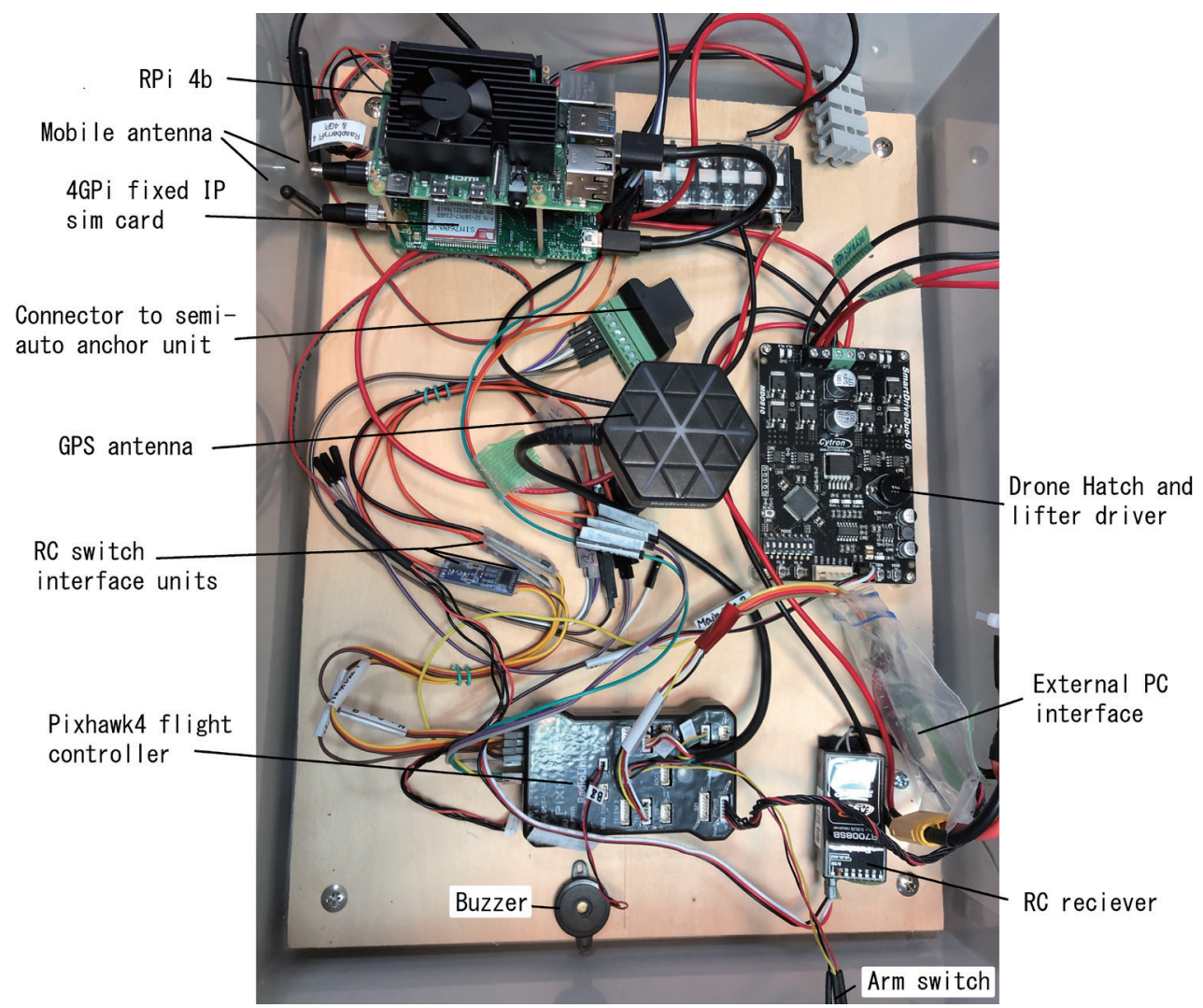

Fig. 3. (Color online) ASV-DBS main control unit.

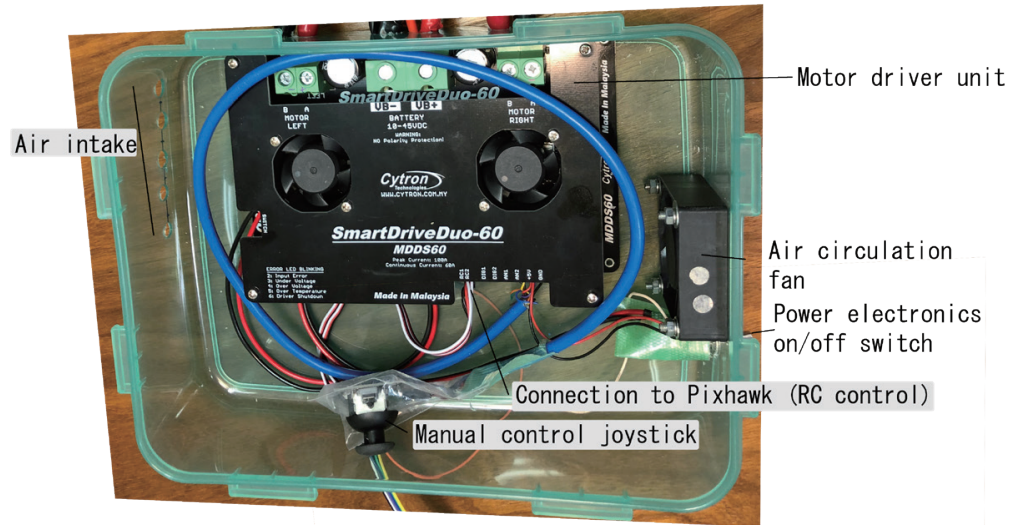

Fig. 4. (Color online) ASV-DBS main motor driver unit.

Figure 4 shows the main motor drive unit, which is housed in a water-resistant container that provides air circulation across the unit. A manual control joystick is available for direct operation of the boat independently of the Pixhawk4 control system. 


\section{Experimental Results}

\subsection{Initial testing on model RC boat}

Initial testing of the Pixhawk4-based control system was, for convenience, carried out using an existing RC boat, shown in Fig. 5. Here, the original RC controller can be seen behind the boat and the Pixhawk4-controlled (air) propellers are visible. The dual control system provides a secure way to retrieve the boat in the case of the test system failing. The initial results of tests at the Isahaya Spectacle Bridge Park can be seen in Ref. 15. The GPS settings screen of the Mission Planner software is shown in Fig. 6. As can be seen in the video in Ref. 15, the RC model boat functioned with $\sim 0.5 \mathrm{~m}$ precision without any modification of the fine parameter settings.

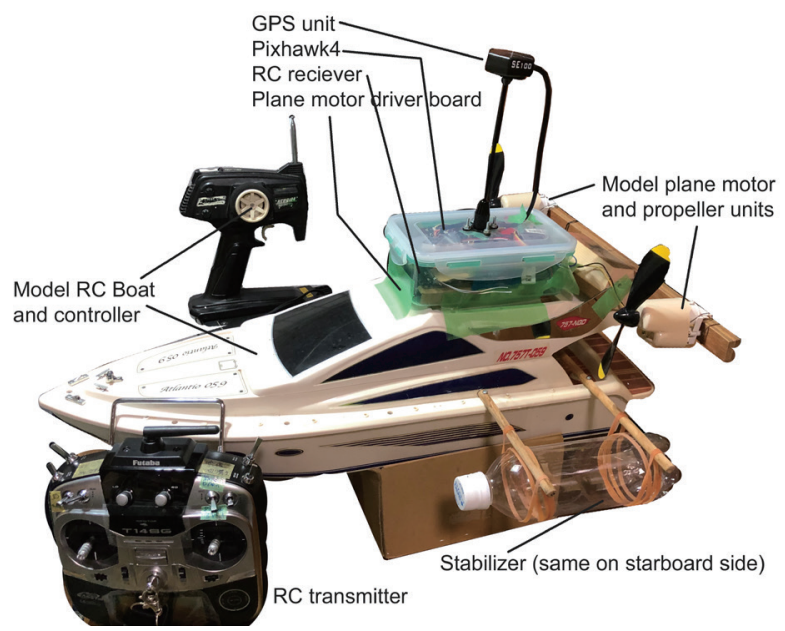

Fig. 5. (Color online) Initial test setup for Pixhawk4-based boat control.

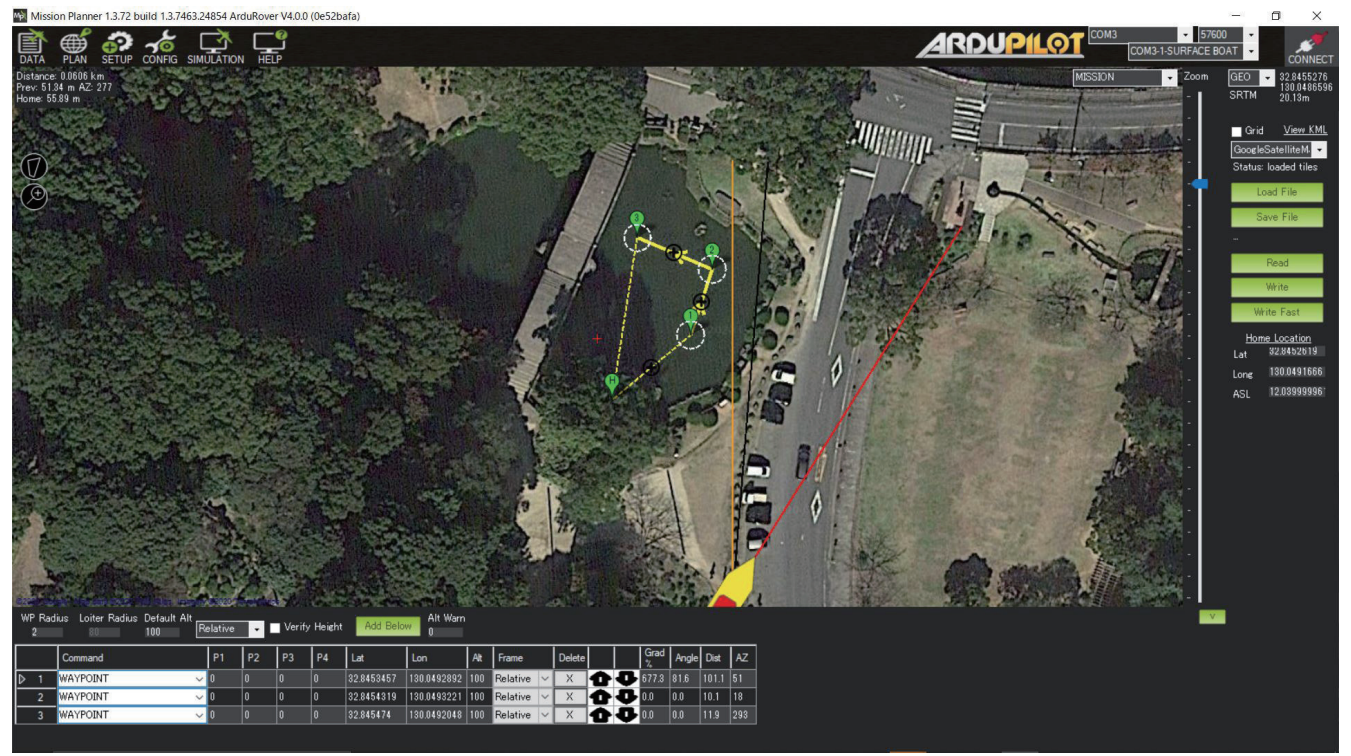

Fig. 6. (Color online) Initial GPS test setup using Mission Planner. 


\subsection{Initial testing of ASV}

The ASV boat with stabilizers was tested in a minimal configuration on the west coast of Tsushima (Koutsuki) in the open sea in April $2020^{(5)}$ before the development and testing of the main control hardware (Pixhawk4). However, when this system was placed in the target ASV boat, significant modifications to the fine parameter settings were required to produce the same navigation precision. The model $\mathrm{RC}$ boat including the additional modifications to house the Pixhawk4 and related equipment weighed about $3 \mathrm{~kg}$, and Pixhawk4 controlled two $6 \mathrm{~W}$ (air) propellers (Fig. 5). For comparison, the ASV weighs about $150 \mathrm{~kg}$ and is powered by two $600 \mathrm{~W}$ (water) propellers (Figs. 1 and 2).

The AUV was tested in Tarami harbor (Isahaya, Nagasaki). Initially, navigation appeared to be random; however, the error was found to exceed the inner harbor area. The boat operated correctly in the outer harbor but the navigation error exceeded $50 \mathrm{~m}$ (as seen in the larger arc traces at the top of Fig. 7). After a day of trial-and-error adjustment of the initial parameters, the same level of precision as the RC boat was achieved. Figure 7 shows the traces (faint purple) of the boat's course during calibration: the final course is shown as a white line and the three waypoints on the white line are shown in green. The specific parameter alterations are shown in Table 2; however, a fundamental mistake in selecting the frame type (set to omni - used for four thrusters) will require this process to be repeated and the corrected settings will be reported at a later date.

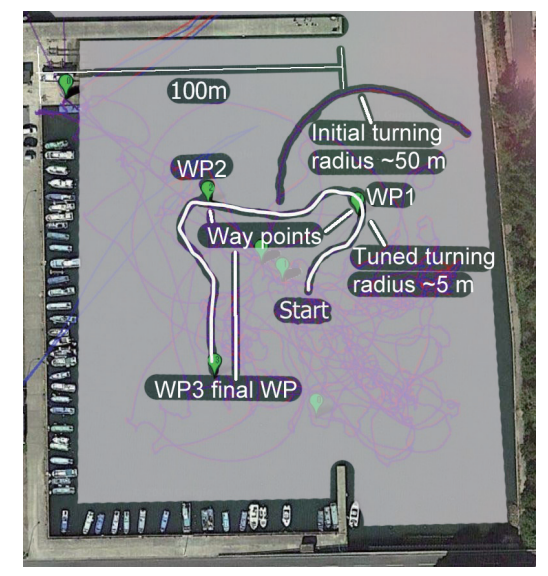

Fig. 7. (Color online) Initial and corrected traces after parameter calibration.

Table 2

Optimized Pixhawk4 steering and navigation parameter settings for ASV.

\begin{tabular}{|c|c|c|c|c|c|}
\hline \multicolumn{3}{|c|}{ Steering rate } & \multicolumn{3}{|l|}{ Navigation } \\
\hline & Default & ASV & & Default & ASV \\
\hline $\mathrm{P}$ & 0.2 & 0.2 & WP Speed & 0.3 & 2.0 \\
\hline $\mathrm{I}$ & 2.0 & 0.2 & WP Radius & 1.0 & 2.0 \\
\hline $\mathrm{D}$ & 0.3 & 0.0 & WP Overshoot & 2.0 & 2.0 \\
\hline IMAX & 1.0 & 1.0 & Turn G Max & 0.1 & 0.6 \\
\hline FF & 2.0 & 0.2 & Lat Acc Cntl Peri & 60 & 8 \\
\hline & & & Lat Acc Cntl Dam & 0.70 & 0.75 \\
\hline
\end{tabular}

P, I, D: proportional, integral, derivative; IMAX: integral error limit (attitude related for flying application); FF: forward feedback; WP: waypoint; Lat Acc Cntl Peri/Dam: lateral acceleration control perimeter/damping 
Parameters that have been changed are highlighted in Table 2. As mentioned above, the frame type was incorrect (omni) and the above parameters will not be correct but will provide some indication of the scale of changes necessary when scaling up the vehicle weight $(\sim 50 \times)$ and power $(\sim 100 \times)$ and changing the propulsion medium from air- to water-driven propellers. Clearly these changes will significantly affect the control dynamics of the vehicle.

The ASV part of the ASV-DBS has been successfully trialed using RC control as well as GPS-based waypoint control using Mission Planner software on a Pixhawk4 hardware platform. The cellular-based control has been successfully bench-tested, demonstrating remote telemetry control of Pixhawk4 from a remote computer running Mission Planner software. The sonar system was tested as a standalone system in a PC on the boat during the Tarami trials (Fig. 7). The power control system incorporating the solar panels visible in Fig. 2 was tested and used during both the Tarami and Shimabara trials.

\section{Discussion}

To date, the sonar has been tested and used independently, but not yet integrated with the Pixhawk4 system, which provides the sonar interface. The next major step is to automate the onboard drone's functionality. This will require an onboard charging system and a precision landing system using GPS coordinates combined with a camera-based system to lock onto the landing bay, ${ }^{(16)}$ as well as integration with the existing Mission-Planner-based control system. Once the entire system is integrated, significant work will be required to provide the maximum level of autonomy possible, so that contact with a human operator is only required when an unexpected situation arises or remote guidance is required into or out of a harbor.

Currently, propellers are used. However, during one test, a stringy type of seaweed was encountered that quickly became entangled in both propellers, bringing the boat to a standstill. Two approaches can be used to overcome this issue: either use vertical-intake-based propulsion or provide full housing for the propellers, and upon the detection of blocked intakes, reverse the motors to clear the blockage.

The current configuration is designed with a significant wave height (SWH) of about $2 \mathrm{~m}$ in mind. As an experimental unit, the drone bay is fully functional. However, the drone bay is "water-resistant" but not "watertight." Clearly, for practical use, the ASV must be able to withstand temporary submersion, and therefore the deck must be watertight. All systems above the deck, i.e., the cameras, anchor winch, and weather monitoring system, must be resilient to temporary submersion. ${ }^{(17)}$ The camera will also require a means of internal defogging as well as an external cleaning mechanism; as mentioned previously, the main function of the onboard camera is to facilitate the remote control of navigation into harbors.

\section{Conclusion}

The concept of an ASV-DBS is gradually nearing reality. The basic operation of the ASV has been confirmed to be seaworthy and controllable by RC operation and operable using preprogrammed waypoints set up using Mission Planner flight control software. Full remote 
control using a mobile phone interface has been tested in the laboratory and is expected to be trialed in the near future.

\section{Acknowledgments}

This work was supported by an initiative called the Ikkaku (Sea Unicorn) project, funded by The Nippon Foundation; Japan Advanced Science and Technology Organization for Education, Human Resources and Research (JASTO); and Leave a Nest Co., Ltd., as part of a team initially referred to as CCSC (Coastal Cleanup using Satellites and Drones) and more recently renamed "Debris Watchers" (team manager: Yu Kudo of Amanogi Corp.). Thanks go to students and staff in the Yamamoto Lab., to Tomoyuki Sato (System Five Co., Ltd.), to Takaaki Morita (Nagasaki prefecture) for project planning, to System Five Co., Ltd., for the development of the cellular control interface, to Production NAP for drone videography and assistance in the Tarami trials, to Yasunori Izumi (Musokagaku, Co., Ltd.) for guidance in the use of Pixhawk4, RC-related matters, and the Shimabara trial, to Eito Matsuura (Nag. Uni. Dept. of Fisheries student) for assistance with calibration in Tarami harbor, to Shintaro Uryu (Nag. Uni. Dept. of Fisheries student), Ren Ota (Nag. Uni.), and Kenji Kumamoto (local fisherman at Koushiki) for assistance in the Koushiki Tsushima trials, and to the harbormasters in Koutsuki (Tsushima Island), Shimabara, and Tarami (Isahaya), Nagasaki, for their cooperation in this research.

\section{References}

1 Flytbase: Drone Automation Solution for Security \& Surveillance: https://flytbase.com/drone-securitysolution/ (accessed December 2020).

2 Azur Drones: https://www.azurdrones.com/remote-monitoring-by-drone/ (accessed December 2020).

3 Quaternium: Hybrid Drones, UAVs: https://www.quaternium.com/ (accessed December 2020).

4 Nippon.com: Spoiled Shores: Japan's Tsushima on the Front Line of Marine Plastics: https:/www.nippon.com/ en/japan-topics/c06301/spoiled-shores-japan $\% \mathrm{E} 2 \% 80 \% 99$ s-tsushima-on-the-front-line-of-marine-plastics. html (accessed December 2020).

5 Nagasaki BC: ASV-DBS (Autonomous Surface Vessel - Drone Base Station) Trials in Koshiki Tsushima and Shimabara: https://youtu.be/Gait4Mud sQ (accessed December 2020).

6 Nagasaki BC: ASV DBS (Autonomous Surface Vessel, Drone Base Station) Hatch and Lifter Mechanism: https://youtu.be/GlyjId9mgN4 (accessed December 2020).

7 UAVMatrix: UAVcast-Pro: https://uavmatrix.com/product/uavcast-pro/ (accessed December 2020).

8 Mission Planner, ArduPilot: https://ardupilot.org/planner/ (accessed December 2020).

9 Blue Robotics: Ping Sonar Altimeter and Echosounder, https://bluerobotics.com/store/sensors-sonars-cameras/ sonar/ping-sonar-r2-rp/ (accessed December 2020).

10 Wikipedia, Tsushima Island: https://en.wikipedia.org/wiki/Tsushima_Island (accessed December 2020).

11 Autonomous Control Systems Laboratory Ltd.: https://www.acsl.co.jp/ (accessed December 2020).

12 Ridge-I, (AI): https://ridge-i.com/ (in Japanese) (accessed December 2020).

13 Production NAP (Nagasaki): http://www.production-nap.com/ (accessed December 2020) (in Japanese).

14 J. Han, Y. Cho, J. Kim, J. Kim, N.-S. Son, and S. Y. Kim: J. Field Robot. 37 (2020) 987. https://doi.org/10.1002/ rob.21935

15 Nagasaki BC: Pixhawk GPS Based Controller Test at Isahaya Park: https://youtu.be/kR25KaAe9ms (accessed December 2020).

16 Flytbase: Precision Landing System: https://flytbase.com/precision-landing/ (accessed December 2020).

17 ISO: Small Craft — Stability and Buoyancy Assessment and Categorization — Part 3: Boats of Hull Length Less Than $6 \mathrm{~m}$ : https://www.iso.org/obp/ui/\#iso:std:iso:12217:-3:ed-3:v1:en (accessed December 2020). 